\title{
SOLAR NEIGHBOURHOOD AS THE LOCAL MACROSCOPIC VOLUME ELEMENT WITHIN THE GALAXY
}

\author{
T. A. AGEKJAN and K. F. OGORODNIKOV \\ Dept. of Astronomy, University of Leningrad, U.S.S.R.
}

The notion of the local Macroscopic Volume Element (MVE) is introduced in order to facilitate the application of hydro-dynamical methods of treating internal motions in stellar systems and in particular in our Galaxy. The MVE must characterize the properties of the stellar system in a given point within the system. Hence the diameter of the MVE must be taken as small as possible; it must, however, contain a sufficiently large number of stars in order that the laws of statistics might be applied. The greater is the local star density the smaller can be made the size of the MVE.

Modern catalogues of nearby stars by Gliese (1969) and Woolley et al. (1970) afford a good collection of data for a determination of local characteristics of the Galaxy in the immediate neighbourhood of the Sun. In the first section we discuss such characteristics as the local stellar density, the local luminosity function and the influence of observational selection upon them. In the second section we consider the local solar motion and the effect of the asymmetry of stellar motions. The responsibility for the content of paragraph 1 lies primarily upon T. A. Agekjan while that for paragraph 2 upon K. F. Ogorodnikov. We both are indebted to G. V. Ishkhanov and to L. V. Chuvicova for substantial help in numerical computations.

(1) The stellar density, the proportion of double and multiple stars and the luminosity function of stars were determined using the catalogue of nearest stars by Woolley et al. (1970).

The method of extrapolation to zero distance was applied. In this method several overlapping concentric spherical layers within the sphere around the Sun are considered and the mean density of registered catalogue objects $D^{*}\left(r_{i}\right)$ in each spherical layer is considered.

$r_{i}$ is the mean distance of the $i$ th spherical layer from the center.

The solution of the equations

$$
a+b r_{i}+c r_{i}^{2}=D^{*}\left(r_{i}\right)
$$

by the method of least squares gives

$$
a=D^{*}(0)=D
$$

the true density of objects in the neighbourhood of the Sun.

In our calculations 22 overlapping spherical layers were considered in the sphere of radius $25 \mathrm{pc}$, the common part of two adjacent layers being $3 \mathrm{pc}$ thick.

The results are:

(a) The mean stellar density in the neighbourhood of the Sun is equal to

$$
D=0.138 \pm 0.009 \mathrm{stars}^{-3} \text {. }
$$


(b) The proportion of stars which are components of double and multiple systems among all stars around the Sun is equal to

$$
0.73 \pm 0.04
$$

(c) All the stars were divided in eight groups according to their absolute magnitudes. The mean density of the stars of each individual group was calculated by means of Equation (1). Table I shows the proportions of stars in each interval of absolute magnitude among all stars population.

TABLE I

Luminosity function

\begin{tabular}{ll}
\hline$M$ & \multicolumn{1}{l}{$\Delta$} \\
\hline$-1.0 \leqslant M \leqslant+4.6$ & $0.0052 \pm 0.0008$ \\
$+4.5<M \leqslant+6.5$ & $0.0107 \pm 0.0008$ \\
$+6.5<M \leqslant+8.5$ & $0.0088 \pm 0.0007$ \\
$+8.5<M \leqslant+10.0$ & $0.0079 \pm 0.0008$ \\
$+10.0<M \leqslant+11.5$ & $0.0237 \pm 0.0011$ \\
$+11.5<M \leqslant+13.5$ & $0.0254 \pm 0.0029$ \\
$+13.5<M \leqslant+15.5$ & $0.0112 \pm 0.0012$ \\
$+15.5<M \leqslant+19.5$ & $0.0071 \pm 0.0009$ \\
\hline
\end{tabular}

The corresponding luminosity function has the appearance shown in Figure 1.

(2) The solar motion ordinarily determined from motions of stars brighter than some given visual magnitude has little sense, if any, from the point of view of stellar dynamics since it is based practically upon stars of large absolute brightness only and thus disregards the multitude of dwarf stars which really constitute the main content of the solar surroundings, i.e. the MVE. In order to make first steps in clearing up the situation we determined the linear galactic components $u_{0}, v_{0}, w_{0}$ of the solar motion. These quantities were computed for 25 concentric spherical volumes with radii ranging from 10 to $25 \mathrm{pc}$. The results are shown in Figure 2. The total number of stars used is 1361 (for $r=25 \mathrm{pc}$ ). The smallest of the spheres has the radius equal to $10 \mathrm{pc}$ and contains 202 stars. The intermediate radii in the average differ from the adjacent ones by approximately $0.6 \mathrm{pc}$ but in fact the radii were allowed to vary in order that the increase of the number of stars in adjacent regions would be more or less uniform. It appears from Figure 2 that with increasing $r$ the values of $u_{0}$ and $v_{0}$ are steadily increasing while those of $w_{0}$ are decreasing.

This phenomenon is not easy to explain. It may be suggested that it is due to the asymmetry of stellar motions. It is now generally accepted beginning with classical works of Strömberg and Oort that high velocity stars are those which move along very elongated galactic orbits and belong to Population II. This effect is more clearly seen in the $v_{0}$ and $w_{0}$ components. The larger is the spherical volume the greater is the percentage of Population II stars within it since, roughly speaking, the latter rises proportionally to $r^{3}$ while the number of the 'flat' Population I stars rises only as $r^{2}$. 


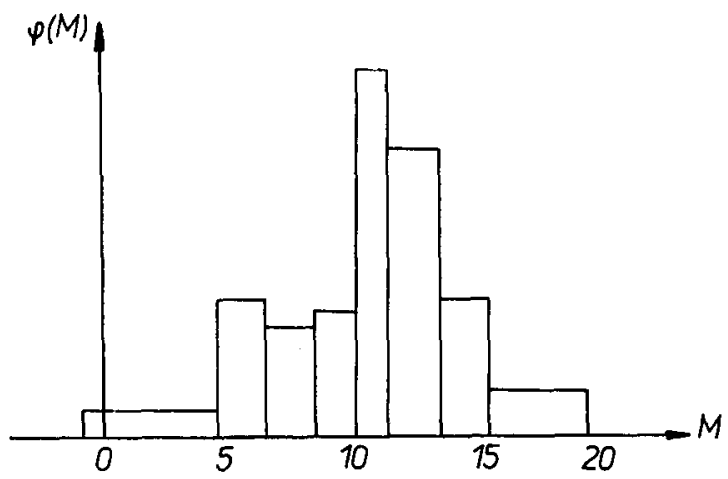

Fig. 1. The luminosity function.

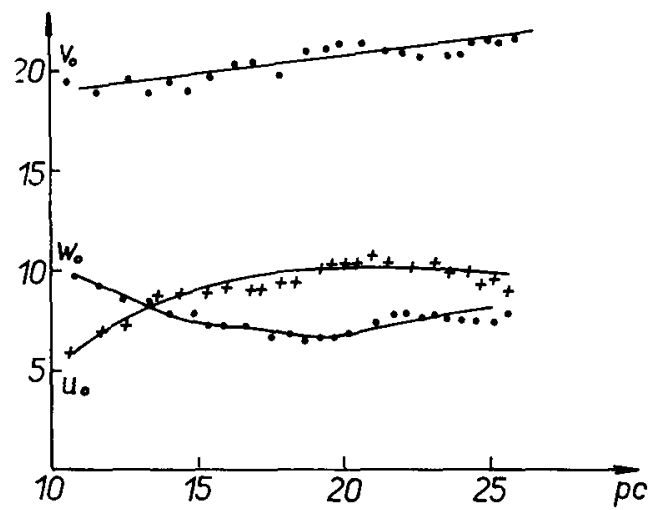

Fig. 2. Velocity components dependent on the radius of the spherical volume considered.

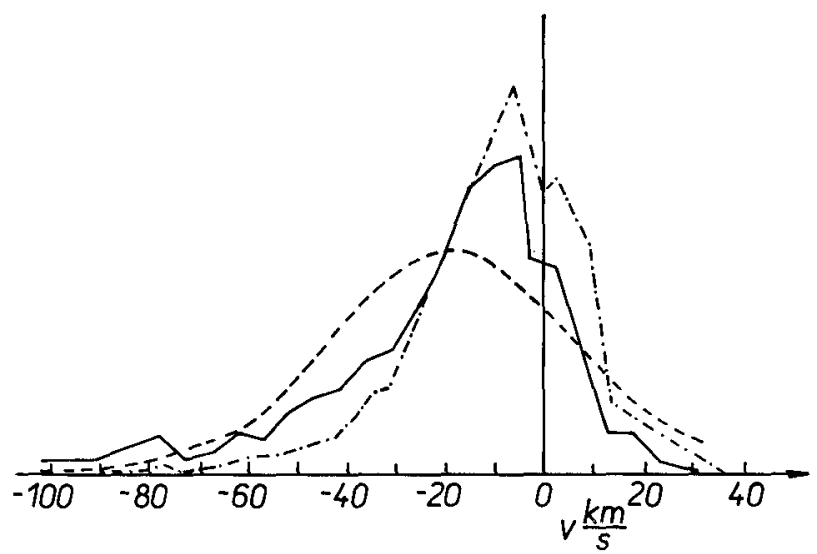

Fig. 3. Distribution of the $v$-component. Observed (full), best fitting Gaussian (dash), and the weighted distribution (dot-dash). 
In the $v_{0}$ component the Population II stars lag behind the Population $\mathbf{I}$ and thus increase the solar velocity. This we shall consider in some detail later on. On the contrary, the surplus of Population II stars makes the $w_{0}$ component smaller since the elongated orbits which happen to pass through the solar neighbourhood must have preferentially orbits with small inclinations towards the galactic plane, i.e. small $w$-components. The increase in the $u_{0}$ component perhaps is due to a minor asymmetry of stellar motions in projection to this direction. This should have been expected apriori since the axis of Strömberg's asymmetry as determined by himself makes an angle of 6.5 with the adopted $v$-direction. Of course all these remarks are of qualitative character only and require a more detailed consideration.

The stellar velocity components most strongly affected by the asymmetry are the $v$-components. This is clearly seen in Figure 3 where the distribution curve of $v$ components of 1355 stars of the catalogue by Woolley et al. is plotted (full line). It is seen that at the positive end the curve sharply drops to zero at $v=35-40 \mathrm{~km} \mathrm{~s}^{-1}$ while toward the negative side the curve streches up to $-125 \mathrm{~km} \mathrm{~s}^{-1}$ and there are a few stars (12 in number) much farther off, up to more than $-400 \mathrm{~km} \mathrm{~s}^{-1}$, while at the positive side there is not a single star. This is quite in accordance with Oort's high velocity star phenomenon.

The ordinary procedure with assigning equal weights to all stars, except 12 'discordant' ones which were rejected, gives $v_{0}=19.6 \mathrm{~km} \mathrm{~s}^{-1}$ and $\sigma=24.4 \mathrm{~km} \mathrm{~s}^{-1}$. The dashed smooth curve represents the best fitting Gaussian with the same values of the three parameters as the observed (full) curve.

The ordinary procedure of deriving the solar motion (this time $v$ ) is equivalent to assigning equal weights $p=1$ to all stars except the discordant ones to whom we assign the weight $p=0$. The difference between the observed curve and the Gaussian is marked. The observed curve shows, besides the asymmetry, a substantial positive excess which is an indication of a statistical non-homogeneity of the stellar ensemble. A more rational method of assigning weights may be the following one (s.f. Ogorodnikov, 1928).

We put the observed distribution function of $F(v)$ to be a superposition of Gaussians with different centers and dispersions in the form

$$
F(v)=\frac{N}{\pi^{1 / 2}} \int_{0}^{\infty} f(h) h e^{-h^{2}\left(v-v_{h}\right)^{2}} \mathrm{~d} h,
$$

where $h^{2}=\frac{1}{2} \sigma^{-2}$ and $v_{h}$ is a partial centroid of the particular Gaussian which depends upon its value of $h . f(h)$ is an unknown normalized distribution function of the $h$ values. We then easily have that

$$
\varphi(v)=-\frac{F^{\prime}(v)}{2 F(v)}=\overline{h^{2}(v)}\left[v-\overline{v_{h}(v)}\right],
$$

where 


$$
\begin{aligned}
\overline{h^{2}(v)}= & \frac{\int_{0}^{\infty} f(h) h^{3} \exp \left[h^{2}\left(v-v_{h}\right)^{2}\right] \mathrm{d} h}{\int_{0}^{\infty} f(h) h \exp \left[h^{2}\left(v-v_{h}\right)^{2}\right] \mathrm{d} h} \\
\overline{v_{h}(v)}= & \frac{\int_{0}^{\infty} f(h) v_{h} h^{3} \exp \left[h^{2}\left(v-v_{h}\right)^{2}\right] \mathrm{d} h}{\int_{0}^{\infty} f(h) h^{3} \exp \left[h^{2}\left(v-v_{h}\right)^{2}\right] \mathrm{d} h}
\end{aligned}
$$

are respectively the average weight and the weighted mean value of $v_{h}$ corresponding to all stars of the ensemble which have the same velocity $v$.

But following Strömberg (1923) we may write

$$
v_{h}=a h^{-2}+b
$$

where $a=-0.0096 \mathrm{~s} \mathrm{~km}^{-1} ; b=-10.0 \mathrm{~km} \mathrm{~s}^{-1}$ are Strömberg's constants. Substituting (4) into the formula for $\overline{v_{h}(v)}$ we get

$$
\overline{v_{h}(v)}=a\left[\overline{h^{2}(v)}\right]^{-1}+b
$$

and then using (2)

$$
\begin{aligned}
\overline{h^{2}(v)} & =\frac{a-\varphi(v)}{v-b}, \\
\overline{v_{h}(v)} & =\frac{a v-b \varphi(v)}{a-\varphi(v)}
\end{aligned}
$$

From (4) it is seen that for $h=\infty v_{h}=b$. But $h=\infty$ means that $\sigma$ the dispersion of peculiar stellar velocities in this particular subgroup is zero. Thus $b$ is equal to velocity relatively to the Sun of mass points. From the second Equation (6) we see that $\overline{v_{h}(b)}=b$. It seems that $v=b$ is a point of discontinuity for $\overline{h^{2}(v)}$. But this is not so since it is easily shown that $\varphi(b)=a$ and the indetermination is avoided by using de l'Hospital rule. The above weights are decreasing rather rapidly for large $v$. For instance they drop to less than 0.05 of the maximum value for $v=-60$ and $v=+30$ $\mathrm{km} \mathrm{s}^{-1}$. In Figure 3 the dot-dash curve represents the weighted distribution of the $v$-component velocities subject to the condition that the total number of stars remains the same, i.e. $N=1355$. The weighted solar velocity appears to be $v_{0}=11.7$ and $\sigma=$ $=18.7 \mathrm{~km} \mathrm{~s}^{-1}$, i.e. both are substantially smaller than with equal weights. 


\section{References}

Gliese, W.: 1969, Veröffentl. Astron. Rechen-Inst. Heidelberg, Nr. 22.

Ogorodnikov, K. F.: 1928, Astron. Zh. 1, 1.

Strömberg, G.: 1923, Astrophys. J. 59, 229.

Woolley, R., Epps, E. A., Penston, M. J., and Pocock, S. B.: 1970, Roy. Obs. Ann. No 5.

\section{DISCUSSION}

Perek: How does the derived value of the density of 0.138 compare with the value derived by you, Dr Wielen?

Wielen: For stars in Gliese's Catalogue it would be about $\mathbf{0 . 1 1}$, that is in a good agreement.

Gliese: The region around $25 \mathrm{pc}$ is sensitive to the accuracy of parallaxes. We must keep in mind that positive errors are preferred. I assume that the authors have tried to eliminate this effect. 